\title{
Structure determination of amorphous molecular solids by NMR crystallography
}

\section{Lyndon Emsley}

\author{
EPFL, Lausanne, Switzerland;
}

lyndon.emsley@epfl.ch

Structure elucidation of amorphous molecular solids presents one of the key challenges in chemistry today. Knowledge of the atomiclevel structure in such materials would be of great value for example to direct the optimisation of pharmaceutical formulations. However, the lack of long-range structural order prevents atomic-level characterization of these materials using methods like single crystal X-Ray diffraction. Solid state NMR on the other hand yields atomic-level information on amorphous materials and molecular dynamics (MD) can generate candidate sets of possible disordered structures. Directly relating these two techniques has been out of reach so far, due to the prohibitive cost of computing chemical shifts on large ensembles of large MD structures using DFT. Recently, a method based on machine learning, dubbed ShiftML (1), has emerged as a quick and accurate way to predict the chemical shifts of organic solids, even for large systems.

Here, using a machine learning model of chemical shifts, we determine the complete atomic-level structure of the amorphous form of a drug molecule by combining dynamic nuclear polarization-enhanced solid-state NMR experiments with predicted chemical shifts for MD simulations of large systems (2). From these amorphous structures we then identify H-bonding motifs and relate them to local intermolecular interaction energies.

1. Paruzzo, F. M.; Hofstetter, A.; Musil, F.; De, S.; Ceriotti, M.; Emsley, L., "Chemical shifts in molecular solids by machine learning." Nat Commun 2018, 9, 4501. doi.org/10.1038/s41467-018-06972-x

2. Cordova, Balodis, Hofstetter, Paruzzo, Nilsson Lill, Eriksson, Berruyer, Simões de Almeida, Quayle, Norberg, Svensk Ankarberg, Schantz, Emsley, "Structure determination of an amorphous drug through large-scale NMR predictions." Nat Commun 2021, doi.org/10.1038/s41467021-23208-7

Keywords: solid-state NMR, amorphous molecular solids 